\title{
WIDTH-INTEGRALS OF CONVEX BODIES
}

\author{
ERWIN LUTWAK
}

ABSTRACT. The width-integrals (Breitenintegrale) as introduced by Blaschke are examined. They are shown to satisfy a cyclic inequality similar to that satisfied by the cross-sectional measures (Quermassintegrale). Other similarities and inequalities between the width-integrals and the cross-sectional measures are shown to exist.

The setting for this paper is Euclidean $n$-space $R^{n}$. Compact convex sets with nonempty interiors are called convex bodies and the space of all convex bodies endowed with the Hausdorff topology is denoted by $\mathcal{K}_{n}$. We denote the unit $n$-ball and the unit $(n-1)$-sphere by $U$ and $\Omega$, respectively. For a convex body $K$, we use $W_{i}(K)$ to denote its cross-sectional measure (Quermassintegral) of index $i$. The $n$-dimensional volume of $K$ is written as $V(K)$. For $\omega \in \Omega, b_{K}(\omega)$ is defined to be half the width of $K$ in the direction $\omega$. Two bodies $K$ and $L$ are said to have similar width if there exists a constant $\lambda>0$ such that $b_{K}(\omega)=\lambda b_{L}(\omega)$ for all $\omega \in \Omega$. For $K \in \mathcal{K}_{n}$ and $p \in$ int $K$, we use $K^{p}$ to denote the polar reciprocal of $K$ with respect to the unit sphere centered at $p$. For reference see Bonnesen and Fenchel [2] and Hadwiger [5].

Width-integrals (Breitenintegrale) were first considered by Blaschke $[1$, p. 85] and later by Hadwiger [5, p. 266]. The width-integral of index $i$ is defined by:

\section{Definition.}

$$
B_{i}(K)=\frac{1}{n} \int_{\Omega} b_{K}^{n-i}(\omega) d S(\omega) \quad\left[i \in R ; K \in K_{n}\right]
$$

where $d S$ is the $(n-1)$-dimensional volume element on $\Omega$.

We note that our definition differs slightly from that of Blaschke in that we multiply by suitable constants to normalize the $B_{i}$ 's. In particular, we have $B_{i}(U)=\omega_{n}$ for all $i \in R$ and $B_{n}(K)=\omega_{n}$ for all $K \in \mathcal{K}_{n}$, where $\omega_{n}$ denotes the volume of the unit $n$-ball.

The width-integral of index $i$ is a map,

$$
B_{i}: K_{n} \rightarrow R
$$

It is positive, continuous, homogeneous of degree $n-i$ and invariant under

Received by the editors May 2, 1974 and, in revised form, June 28, 1974.

AMS (MOS) subject classifications (1970). Primary 52A40, 52A20.

Key words and phrases. Cross-sectional measures, Hölder's Inequality, Minkowski's Inequality, polar body, support function, width, width-integrals. 
motions. In addition, for $i \leq n$ it is also bounded and monotone under set inclusion.

The following simple consequence of Hölder's Inequality [6, p. 140] will be required to prove our main theorem.

Lemma 1. If $f$ and $g$ are nonzero powers of a (strictly) positive continuous function $\phi$ defined on $\Omega$ and $p>1$, then

$$
\left[\int_{\Omega} f(\omega) g(\omega) d S(\omega)\right]^{p} \leq\left[\int_{\Omega} f^{p}(\omega) g(\omega) d S(\omega)\right]\left[\int_{\Omega} g(\omega)^{p} d S(\omega)\right]^{p-1}
$$

with equality if and only if $\phi$ is constant on $\Omega$.

The cross-sectional measures satisfy the cyclic inequality [5, p. 282]:

$$
W_{i}^{k-j}(K) W_{k}^{j-i}(K) \leq W_{j}^{k-i}(K) \quad\left[i<j<k ; K \in K_{n}\right]
$$

with equality if $K$ is an $n$-ball. The width-integrals satisfy the cyclic inequality given in

\section{Theorem 1.}

$$
B_{j}^{k-i}(K) \leq B_{i}^{k-j}(K) B_{k}^{j-i}(K) \quad\left[i<j<k ; K \in K_{n}\right]
$$

with equality if and only if $K$ is of constant width.

To prove this we simply let $f=b_{K}^{i-j}, g=b_{K}^{n-i}$ and $p=(k-i) /(j-i)$ in Lemma 1.

$$
\text { Lemma 2. } B_{n-1}(K)=W_{n-1}(K) \quad\left[K \in K_{n}\right] .
$$

This is well known [5, pp. 211-212].

\section{Proposition 1.}

$$
W_{i}(K) \leq B_{i}(K) \quad\left[i<n-1 ; K \in K_{n}\right]
$$

with equality if and only if $K$ is an $n$-ball.

Proof. In general, we have (see [5, p. 278]):

$$
W_{i}(K) \leq \omega_{n}^{i+1-n} W_{n-1}^{n-i}(K)
$$

with equality if and only if $K$ is an $n$-ball. From Theorem 1 we have

$$
\omega_{n}^{i+1-n} B_{n-1}^{n-i}(K) \leq B_{i}(K)
$$

with equality if and only if $K$ is of constant width. The desired result is obtained when we combine (1) and (2) using Lemma 2. 
For the cross-sectional measures of the Minkowski sum $K+L$ of two convex bodies $K$ and $L$ we have the following inequality [5, p. 249]:

$$
W_{i}(K+L)^{1 /(n-i)} \geq W_{i}(K)^{1 /(n-i)}+W_{i}(L)^{1 /(n-i)} \quad\left[i<n-1 ; K, L \in \mathcal{K}_{n}\right]
$$

with equality if and only if $K$ and $L$ are homothetic. For the width-integrals of the Minkowski sum of two convex bodies we have

Theorem 2.

$$
B_{i}(K+L)^{1 /(n-i)} \leq B_{i}(K)^{1 /(n-i)}+B_{i}(L)^{1 /(n-i)} \quad\left[i<n-1 ; K, L \in \mathcal{K}_{n}\right]
$$

with equality if and only if $K$ and $L$ have similar width.

To prove this we use Minkowski's Inequality [6, p. 146]'in conjunction with the fact that $b_{K+L}=b_{K}+b_{L}$.

For the cross-sectional measures of the parallel body $K_{\mu}=K+\mu U$ we have the Steiner polynomial expression [5, p. 214]:

$$
W_{j}\left(K_{\mu}\right)=\sum_{i=0}^{n-j}\left(\begin{array}{c}
n-j \\
i
\end{array}\right) W_{j+i}(K) \mu^{i} \quad[j=0,1, \ldots, n ; \mu>0] .
$$

Analogously for the width-integrals of a parallel body we have

Theorem 3.

$$
B_{j}\left(K_{\mu}\right)=\sum_{i=0}^{n-j}\left(\begin{array}{c}
n-j \\
i
\end{array}\right) B_{j+i}(K) \mu^{i} \quad[j=0,1, \ldots, n ; \mu>0] .
$$

To prove this we merely note that $b_{K_{\mu}}=b_{K}+\mu$.

\section{Proposition 2.}

$$
B_{2 n}(K) \leq V\left(K^{p}\right) \quad\left[K \in \mathcal{K}_{n}\right]
$$

with equality if and only if $K$ is symmetric with respect to $p$.

Proof. Let $H_{K}$ be the support function of $K$ for the origin $p$. We apply Minkowski's Inequality [6, p. 146] to

$$
B_{2 n}(K)^{-1 / n}=\left[\frac{1}{n} \int_{\Omega}\left[2^{-1} H_{K}(\omega)+2^{-1} H_{K}(-\omega)\right]^{-n} d S(\omega)\right]^{-1 / n}
$$

and obtain

$$
B_{2 n}(K) \leq \frac{1}{n} \int_{\Omega} H_{K}^{-n}(\omega) d S(\omega)
$$

with equality if and only if $H_{K}(\omega)=H_{K}(-\omega)$ for all $\omega \in \Omega$. This is the desired result.

As an immediate corollary to. Proposition. 2 we have a simple proof of 
Corollary. If $K$ is a convex body symmetric with respect to $c \in$ int $K$, then $\operatorname{Inf}\left\{V\left(K^{p}\right) \mid p \in\right.$ int $\left.K\right\}=V\left(K^{c}\right)$.

We note that this result is a simple consequence of a general (but not easily proven) theorem of $\mathrm{H}$. Guggenheimer [4].

If, for $K \in K_{n}$, we let $F(K)=B_{2 n}(K) \operatorname{Sup} V^{-1}\left(K^{p}\right)$, where the supremum is taken over all $p \in$ int $K$, then Proposition 2 (in conjunction with earlier comments) can be used to show that $F$ is a similarity invariant measure of symmetry as defined by B. Grünbaum [3, p. 234].

Proposition 3.

$$
B_{n+i}(K) \leq W_{n-i}\left(K^{p}\right) \quad\left[0<i<n ; K \in \mathcal{K}_{n}\right]
$$

with equality if and only if $K$ is an $n$-ball and $p$ is its center.

Proof. In general, we have (see [5, p. 278])

$$
\omega_{n}^{n-i} V^{i}\left(K^{p}\right) \leq W_{n-i}^{n}\left(K^{p}\right)
$$

with equality if and only if $K^{p}$ is an $n$-ball. From Theorem 1 we have

$$
B_{n+i}^{n}(K) \leq \omega_{n}^{n-i} B_{2 n}^{i}(K)
$$

with equality if and only if $K$ is of constant width. The desired result is obtained when we combine (1) and (2) using Proposition 2.

The following theorem was previously obtained by the author [7] as in application of inequalities between dual mixed volumes.

\section{Theorem 4.}

$$
\omega_{n}^{n+1-i} \leq W_{n-1}^{n-i}(K) W_{i}\left(K^{p}\right) \quad\left[0 \leq i<n ; K \in K_{n}\right]
$$

with equality if and only if $K$ is an $n$-ball and $p$ is its center.

Proof. We combine Theorem 1 and Lemma 2 to obtain

$$
\omega_{n}^{n+1-i} \leq W_{n-1}^{n-i}(K) B_{2 n-i}(K)
$$

with equality if and only if $K$ is of constant width. If $i>0$, Proposition 3 states

$$
B_{2 n-i}(K) \leq W_{i}\left(K^{p}\right)
$$

with equality if and only if $K$ is an $n$-ball and $p$ is its center. If $i=0$, Proposition 2 states

$$
B_{2 n-i}(K) \leq W_{i}\left(K^{p}\right)
$$


with equality if and only if $K$ is symmetric with respect to $p$. The desired result is obtained when we combine (1) and (2) or (1) and ( $\left.2^{\prime}\right)$ if $i=0$.

\section{REFERENCES}

1. W. Blaschke, Vorlesungen über Integralgeometrie. I, II, Teubner, Leipzig, 1936, 1937; reprint, Chelsea, New York, 1949.

2. T. Bonnesen and W. Fenchel, Theorie der konvexen Körpern, Springer, Berlin, 1934; reprint, Chelsea, New York, 1948.

3. B. Grünbaum, Measures of symmetry for convex sets, Proc. Sympos. Pure Math., Vol. 7, Amer. Math. Soc., Providence, R. I., 1963, pp. 233-270. MR 27 \#6187.

4. H. Guggenheimer, Polar reciprocal convex bodies, Israel J. Math. 14 (1973), 309-316. MR 47 \#9421.

5. H. Hadwiger, Vorlesungen über Inhalt, Oberfläche und Isoperimetrie, Springer, Berlin, 1957.

6. G. H. Hardy, J. E. Littlewood and G. Pólya, Inequalities, Cambridge Univ. Press, London, 1934.

7. E. Lutwak, Dual mixed volumes, Pacific J. Math. 58 (1975), 531-538.

COLUMBIA UNIVERSITY, NEW YORK, NEW YORK 